\title{
Exploiting spatial dimension in cognitive radios and networks
}

\author{
Heli Sarvanko, Marko Höyhtyä, Marja Matinmikko and Aarne Mämmelä \\ VTT Technical Research Centre of Finland \\ Oulu, Finland \\ heli.sarvanko@vtt.fi
}

\begin{abstract}
This paper studies the maximum number of users that can be allocated in the same frequency band in case of using beamforming in a transmitter and a receiver by simulations. With the use of smart antennas the spectrum can be used efficiently, and at the same time the quality of service $(\mathrm{QoS})$ of secondary users is increased in comparison with omnidirectional antennas. The results indicate that more users can transmit in the same area while fulfilling QoS constraints. The obtained results regarding spectrum sensing state that beamforming gives a great improvement in comparison with detection with omnidirectional antennas. Beamforming leads to power savings because the transmission power is sent to the wanted direction. In addition, directional antennas decrease the time needed for sensing and therefore more time for secondary transmission is left than with omnidirectional antennas.
\end{abstract}

\section{INTRODUCTION}

Nowadays wireless networks consist of numerous heterogeneous terminals, and in the future the amount of wireless devices and wireless networks will be increasing rapidly. This means that the need for radio spectrum will increase even though the spectrum is already heavily allocated. However, there are many frequency bands that are not efficiently used all the time. This has led to several discussions and attracted a lot of attention on how to find unoccupied frequency bands more efficiently.

Cognitive radios (CR) [1] have an ability to greatly improve spectral efficiency by sensing spectrum holes, and adjusting the system parameters so that they can fluently communicate with other users without causing harmful interference to the primary user. Spectrum sensing [2] is a technique that cognitive radios can use to obtain information on spectrum use without interventions with the primary user (PU). Spectrum sensing is well investigated in the literature, and numerous studies have been proposed to improve the detection performance as well as to decrease delays caused by sensing duration.

A promising possibility to increase spectrum reuse and sensing performance is to exploit spatial dimension with the help of smart antennas and beamforming techniques [3]. The term smart antenna is used for antenna arrays with adaptive beamforming technology. The terms smart and adaptive depict the antenna's ability to adjust its parameters and the radiation pattern toward a prospective user and suitable to the environment in which it operates. Smart antennas offer a great solution for interference suppression as they have an ability to prevent signals from undesirable directions by creating nulls towards interferers. Thus, adaptive antennas have an ability to decrease the bit error rate as well as to increase the maximum number of links that can coexist in the same frequency band in a given area.

Smart antennas have been employed to improve the performance of the CR networks in various ways. Directional spectrum occupancy measurements in [4] have indicated that the spectrum use can vary depending on the measurement direction. Joint power control and beamforming in a secondary CR network have been considered e.g., in [5]-[8]. The aim of this combination is to control the interference towards PU [5], to minimize the total transmitted power of the CR network [6], and to improve the channel capacity between secondary users (SU) [7], [8]. Interference reduction using beamforming has been the objective also in [9]. Furthermore, beamforming has been proposed to increase the quality of service (QoS) of a single SU when coexisting with multiple PUs, subject to the interference constraints on the PUs [10]. Moreover, smart antennas are employed for the direction of arrival estimation for obtaining the location information in [11] and [12]. Generally speaking, smart antennas increase spectrum reuse and reduce interference to PUs by adapting transmission parameters so that the antenna transmission beam does not cause harmful interference toward PUs.

Beamforming is used for several purposes in the CR literature. It brings clear benefits for power saving by sending the transmission power to the wanted direction. However, there are clearly cases that need to be investigated. Beamforming offers solutions for directed transmission as well as directed environment detection and both need careful investigation. How much the inclusion of beamforming increases the maximum number of users of network comparing to a situation with omnidirectional antennas? Network capacity for an ad hoc network with omnidirectional antennas has been studied in [13]. How sensing performance changes when smart antennas are deployed? Can we actually save energy in the sensing made with smart antennas? There do not seem to be any studies where the maximum number of links for CR network have been investigated. In addition, there are only a limited number of studies on sensing improvement when using beamforming,

This work has been performed in the framework of the COGNAC project, which is partly funded by Finnish Funding Agency of Technology and

Innovation (Tekes) (decision number 40028/08), and the SMAS project, which is partly funded by Academy of Finland (decision number 134624). 
e.g. [14], [15] where cyclostationary and generalised likelihood ratio detectors have been studied.

We have made investigations to answer the previous questions. We have made simulations to find out how much the maximum number of users can be increased by using beamforming techniques compared to the case of using omnidirectinal antennas. Sensing performance simulations have been made to find improvement in probability of detection when beamforming is used in sensing. In contrast to previous studies we consider the use of Welch's periodogram and beamforming together.

The organization of the paper is as follows: Section II shows the system models used in the simulations; Section III presents our numerical results; Section IV concludes the paper.

\section{SYSTEM MODEL}

\section{A. Beamforming in secondary users' communications}

In a cognitive radio system, it has to be assured that there is no interference caused to PU, and PU can operate without interruption and degradation of their QoS. In addition, the QoS for SU has to be guaranteed. We aim to assure certain level of the maximum signal-to-interference-plus-noise ratio (SINR) for all SU receivers. We investigate the maximum number of links that can coexist in the same area while maintaining the SINR constraint.

In our system model we consider SU transmitters (tx) and receivers (rx) that use beamforming techniques. We consider only unidirectional communications from tx to rx. Our goal is to find the maximum number of links that can coexist in a given frequency band for different beamwidths. The aim is to quantify the gain from using beamforming techniques in terms of how many new users can be admitted to the network compared to omnidirectional antennas. The beamwidth is varied to capture the influence of beamforming on the maximum number of links. The size of the area of interest is $1000 \mathrm{~m} \times 1000 \mathrm{~m}$ and the transmission range $r$ for SU is $100 \mathrm{~m}$ or $10 \mathrm{~m}$. To avoid edge effects the area is made continuous so that when the transmission range of the node exceeds the edge of the area, it is continued in the other side of the area.

Location of a tx-rx pair is chosen as follows. First the tx location is selected randomly then the rx location is chosen arbitrarily from the circumference where tx is located in the centre of the circle so that the distance from the transmitter is $100 \mathrm{~m}$ or $10 \mathrm{~m}$. After the link location has been chosen it is reviewed that a new link will not inflict excess interference to other links' receivers. The SINR at the SU receiver is constrained to $5 \mathrm{~dB}$ to assure SU's QoS. If the new link presents excess interruption or the link's own receiver suffers from interference caused by other transmitters, another location is randomly chosen for the link. If this new location also causes interference to a receiver, or the link's own receiver suffers from interference the transmitter of other links, the location is discarded and a new location is randomly set. This is repeated until a location is found where the interference to other users and in the new receiver remains within the SINR constraint or the search is repeated predefined times which is denoted by $M$.
If an adequate location has not been found after $M$ trials, it is decided that there is no more space for new links and the simulation is completed. The larger $M$ is, the better is the possibility to find a suitable location for the link. On the other hand, large $M$ will consume more simulation time and make simulations exhausting. Another thing that has an effect on the average number of links is the ratio between the link length and the length of one side of the whole area. In these simulations we have used $M=20000$. Fig. 1 depicts how the links are randomly located in the area. Fig. 2 shows not only the links but also the transmitter and receiver beams and the ranges when the antennas are directional. Fig. 3 depicts the transmitter interference range. Because the transmission is only in one direction, the interference beam is shown only for the transmitters.

The carrier frequency $f_{\mathrm{c}}$ is $2 \mathrm{GHz}$. The bandwidth $B$ is 100 $\mathrm{MHz}$ and noise power is

$$
N_{\mathrm{n}}=F k T B
$$

where $F$ stands for the noise factor, $k$ is Boltzmann's constant $1.38 \cdot 10^{-23} \mathrm{~J} / \mathrm{K}$, and $T$ is the absolute temperature in kelvins. When $T$ is $290 \mathrm{~K}, F$ is 3 , and $B$ is $100 \mathrm{MHz}$, we obtain $N_{\mathrm{n}}=$ $1.2 \cdot 10^{-12} \mathrm{~W}$. We assume that the SNR at the receiver is $6 \mathrm{~dB}$ and in absolute values $\mathrm{snr}_{\mathrm{rx}}=3.98$. Using this we can calculate the power at the receiver $P_{\mathrm{rx}}=N_{\mathrm{n}} \cdot \mathrm{snr}_{\mathrm{rx}}=4.7797 \cdot 10^{-12} \mathrm{~W}$. Now we can calculate the transmitted power using Friis's equation

$$
P_{\mathrm{tx}}=\frac{P_{\mathrm{rx}}\left(4 \pi f_{\mathrm{c}} r\right)^{2}}{G_{\mathrm{a}} G_{\mathrm{b}} c^{2}}
$$

where $c$ is the speed of light, and $G_{\mathrm{a}}$ and $G_{\mathrm{b}}$ are the receiver and transmitter gains respectively. Gains are calculated by dividing $360^{\circ}$ by the beamwidth. For instance, when the beamwidth in the tx and rx are $120^{\circ}$, the tx and rx gains are both 3 . Thus we obtain $P_{\mathrm{tx}}$ is $3.73 \cdot 10^{-5} \mathrm{~W}$. We set the interference limit at the victim receiver to be $6 \mathrm{~dB}$ under the noise floor. This means that the maximum allowed degradation for the received SNR is $1 \mathrm{~dB}$. Thus, $I_{\mathrm{dB}}=N_{\mathrm{n}}-6 \mathrm{~dB}=-125 \mathrm{~dB}$ and in absolute values interference power is $I=3.1 \cdot 10^{-13} \mathrm{~W}$. The interference distance $r_{\mathrm{i}}$ is calculated by using Friis's equation

$$
r_{\mathrm{i}}=\sqrt{\frac{P_{\mathrm{tx}} G_{\mathrm{a}} G_{\mathrm{b}}}{I}} \cdot \frac{c}{4 \pi f_{\mathrm{c}}}
$$

and we obtain $r_{\mathrm{i}}=389 \mathrm{~m}$. If $r$ is $10 \mathrm{~m}, r_{\mathrm{i}}$ is $38.9 \mathrm{~m}$.

The theoretical performance in terms of the maximum number of links for the setting described above can be evaluated with a geometrical analysis, which is done as follows. The $1 \mathrm{~km}^{2}$ area is divided by the area of the sector. The radius of the sector is the interference distance and the beamwidth is varied from $10^{\circ}$ to $360^{\circ}$. The geometrical analysis does not consider that antenna beams can overlap although at the simulations the antenna beams can overlap if the SINR constraints are maintained. This causes deviation 


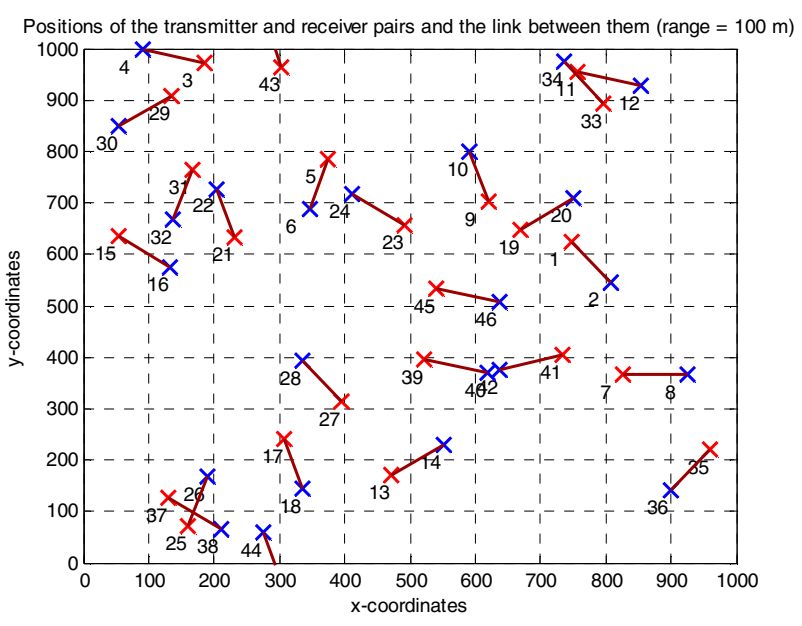

Figure 1. Randomly selected links locations when both tx and rx use directional antennas.

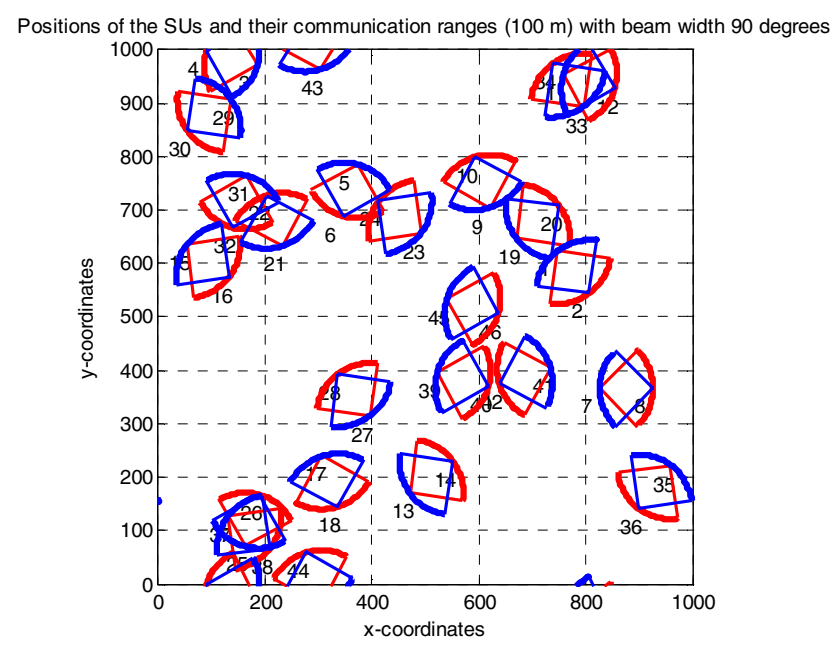

Figure 2. Links and their antenna beams when both tx and rx use directional antennas.

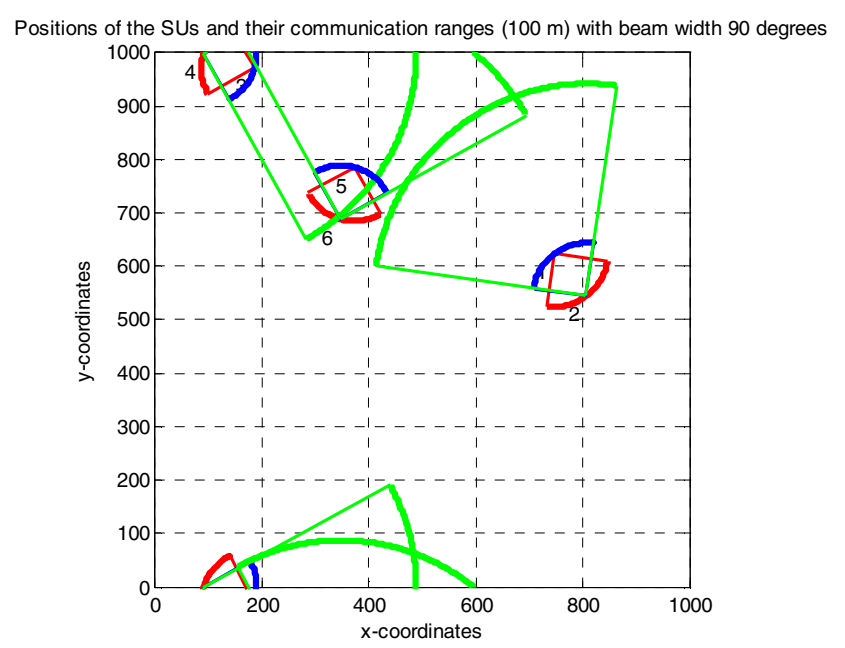

Figure 3. Links and antenna beams and transmitter interference range. between the analysis and simulations since in practice when link places are chosen arbitrarily, antenna beams cannot be placed side by side without gaps. This kind of edge effects will vanish if the size of the area is large compared to link length. However, this brings a bottleneck to the simulations since described method converges slowly.

\section{B. Beamforming in spectrum sensing}

The goal of the spectrum sensing is to decide between the two hypotheses, namely

$$
x(t)=\left\{\begin{array}{cc}
n(t) & , H_{0} \\
h s(t)+n(t) & , H_{1}
\end{array}\right.
$$

where $x(t)$ is the complex signal received by the cognitive radio, $s(t)$ is the transmitted signal of the primary user, $n(t)$ is the additive white Gaussian noise (AWGN) and $h$ is the complex gain of the ideal channel, $H_{0}$ stands for the hypothesis: no signal transmitted, and $H_{1}$ stands for the hypothesis: signal transmitted.

Energy detection is often used for spectrum sensing because of its low complexity. Welch's periodogram is based on energy detection [16]. For Welch's periodogram, the probability of false alarm $P_{\mathrm{f}}$ can be calculated using the central chi-square probability density function (pdf) with $N$ degrees of freedom [17]

$$
P_{\mathrm{f}}=P\left\{Y>\lambda \mid H_{0}\right\}=\frac{\Gamma\left(L M, \frac{\lambda_{\mathrm{t}}}{2 \sigma^{2}}\right)}{\Gamma(L M)}
$$

where $\Gamma(.,$.$) and \Gamma($.$) are the incomplete and complete gamma$ function, respectively, $N$ is the number of degrees of freedom, $\sigma^{2}$ is noise variance, $Y$ is a decision statistic, $\lambda_{\mathrm{t}}$ is the decision threshold, $L$ is the number of frequency bins to be averaged around the zero frequency and $M$ is the number of segments.

The probability of detection $P_{\mathrm{d}}$ can be computed using the noncentral chi-square pdf with $N$ degrees of freedom [17]

$$
P_{\mathrm{d}}=P\left\{Y>\lambda \mid H_{1}\right\}=Q_{L M}\left(\sqrt{\frac{L M A^{2} T}{\sigma^{2}}}, \sqrt{\frac{\lambda_{t}}{\sigma^{2}}}\right)
$$

where $Q_{L M}(.,$.$) is Marcum Q-function, A$ is the signal amplitude, and $T$ is the symbol length. More details can be found from [18]. For simplifying the analysis, we have not taken into account the aliasing phenomenon nor the possible overlapping in Welch's periodogram.

Antenna gain is the maximum directivity gain of an antenna i.e., the directive gain in the direction of the maximum radiation density [19]. Antenna gain is achieved by equally spacing $N_{\mathrm{A}}$ antenna elements in a linear array. Antenna gain $D$ is

$$
D=10 \log \left(N_{\mathrm{A}}\right)
$$


The number of antenna elements has an effect on antenna gain. When the number of the antenna elements increase, the antenna gain increases as well.

\section{SIMULATION RESULTS}

Simulations using the system model from Section II-A have been made with the beamwidth from 10 to 360 degrees. Simulations for each beamwidth have been averaged over 50 simulation cases. In this study only the SU transmitters and receivers are considered. In Fig. 4 the theoretical as well as the simulated average and the maximum number of links for different beamwidth values has been depicted. Both the transmitter and the receiver are using directional antennas. As can be expected, the number of links increases when the beamwidth decreases. We have simulated and analyzed two separate cases, namely link length being either $10 \mathrm{~m}$ or $100 \mathrm{~m}$. For simplicity, the analysis has been made assuming that the beams are not allowed to overlap, although in the simulations the antenna beams can overlap if the SINR constraints are maintained. Thus, analytical results offer a lower bound for the maximum amount of links. The upper bound for the amount of links is unknown.

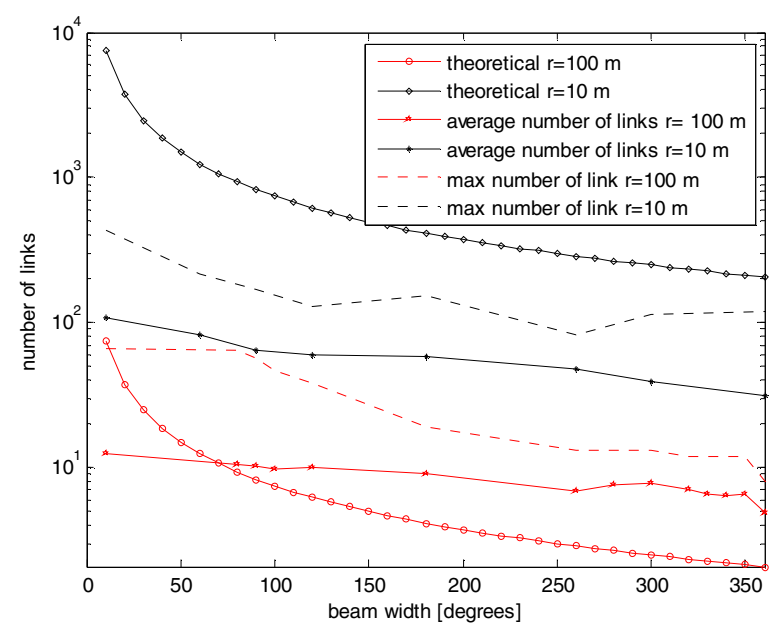

Figure 4. Number of links at the $1 \mathrm{~km}^{2}$ area with different beamwidths.

With a link length of $100 \mathrm{~m}$ the analysis seems to offer a pretty good lower bound for the maximum amount of links. With a link length of $10 \mathrm{~m}$ the simulated results remain under analytical results. This is due to the fact that tx-rx pairs' locations are selected blindly. This leads to a situation where the link places can be repeatedly poor. If the link places are searched for a longer time so that $M>>20000$, the results would approach the real maximum. Unfortunately, this would consume an exceedingly time since the described method converges slowly when $M$ is large. When the link length is short compared to the overall size of the area, the problem with the simulation time is emphasized owing to the increased number of possible links. The results for the link length $100 \mathrm{~m}$ prove that there are more suitable links than what straightforward calculations indicate.
A clear difference can be seen in the slope of the curves. Analytical results drop rapidly when the beamwidth increases. The reduction is much smaller in the simulations. The main reason for the behaviour is in the way the interference is defined. In the simulations interference between users has been taken into consideration by calculating interference distances and interference power between transmitters and receivers using (2) and (3). This is a very practical oriented view of the situation. In theoretical analysis interference between different users has been taken into consideration so that the antenna beams do not overlap. In this kind of scenario theoretical analysis has great challenges since all the practical assumptions are not easily taken into account. It can be seen that a simple analysis could give misleading results. Simulations show that in fact much more links can operate in the same area when compared with analytical curves. Thus, the results clearly indicate the importance of good simulation studies for studying the use of smart antennas in a network level.

For example, with omnidirectional antenna the theoretical curve shows that when the link length is $100 \mathrm{~m}$ only two links are possible to locate in the same area so that they would not cause interference to each other. The simulated curve for link length of $100 \mathrm{~m}$ shows that 8 links could be located in the same area. When the beamwidth is 30 degrees, the theoretical curves show that 24 links could be located in the same area. With a beamwidth of 30 degrees the simulations show that 66 links can be located at the same area. Simulated results also indicate that when the beamwidth decreases from 360 degrees to 30 degrees the number of links increases by $725 \%$.

We made simulations for studying the improvement of spectrum sensing performance using the system model from Section II-B. Beamforming gives also a benefit in power saving by increasing the power in the intended direction while minimizing the power in other directions. With four antenna elements the directivity is $6 \mathrm{~dB}$ whereas 8 and 16 antenna elements give $9 \mathrm{~dB}$ and $12 \mathrm{~dB}$ directivity, respectively.

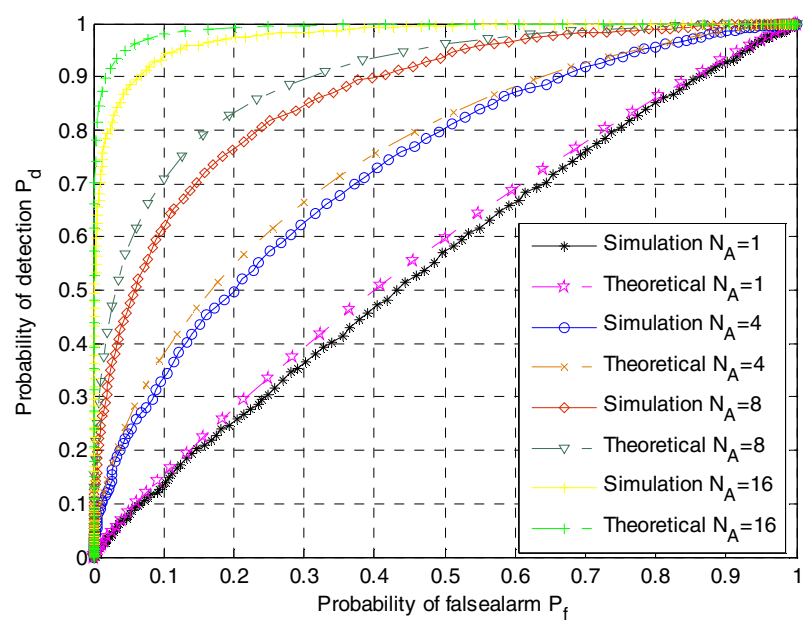

Figure 5. ROC curves for different number of antenna elements.

Fig. 5 presents theoretical and simulated receiver operating characteristic (ROC) curves for Welch periodogram and for a different number of antenna elements. In the simulations we 
have used a complex AWGN channel and QPSK signal. The $\mathrm{SNR}=E / N_{0}$ values are $-15 \mathrm{~dB}$ when there is one antenna element and $-3 \mathrm{~dB}$ when there are 16 antenna elements since directivity gain is $10 \log N_{\mathrm{A}}$. The used FFT length $N_{\mathrm{FFT}}$ is 512 . The FFT length corresponds to the segment length and a rectangular window. We do not use overlapping and block length $N_{\mathrm{b}}$ is 205 symbols. In the analysis and simulations we use $T=4$. In Fig. 5 theoretical results are better than the simulated ones because the theoretical model does not take into account all the phenomena as discussed earlier. In spectrum sensing beamforming improves significantly the probability of detection as can be seen from Fig. 5. This comes from the fact that the smart antenna has a directivity gain which makes the SNR of the sensed signal larger. We can clearly see the performance improvement when we compare the 16 antenna element case to the one antenna element case. When $P_{\mathrm{d}}$ is required to be 0.9 we can see that $P_{\mathrm{f}}$ is clearly below 0.1 when $N_{\mathrm{A}}=16$ whereas $P_{\mathrm{f}}$ is almost 0.9 when $N_{\mathrm{A}}=1$. The latter is absolutely unacceptable because SUs do not have access to spectrum almost at all without causing harmful interference to PUs.

Sensing omnidirectionally the environment with smart antennas requires several rounds of sensing for a certain band. However, when the locations of PUs are known or estimated, SUs can perform sensing towards the locations of transmitters and obtain good sensing results rapidly. From the power saving perspective this is good news. With a higher gain the needed integration time of the sensor is shorter and thus less energy is consumed and more time for the secondary transmission is left.

\section{CONCLUSIONS}

In this paper we have analyzed how many links it is possible to have in the same area when using beamforming. Studies reveal that we can significantly improve the network capacity and secondary network throughput by using directional antennas in the transmission and reception. The results show that the network capacity is increased compared to the omnidirectional antenna case; more users can transmit in the same area while fulfilling QoS constraints. When the beamwidth decreases from 360 degrees to 30 degrees, the number of links increases by $725 \%$. Thus, the results clearly indicate the importance of good simulation studies for studying the use of smart antennas in a network level.

Furthermore, the results demonstrate that the inclusion of smart antennas can be used to improve the performance of cognitive radios in sensing since the probability of detection increases. In addition, when the location of PUs is known, the sensing time can be reduced by directing the sensing beam to the wanted direction. Actually, smart antennas can be seen as an enabling technique for green communications. Power savings are achieved because the transmission power is sent to the wanted direction and the sensing time is decreased.

The next step would be to include primary users in the network and verify that the network will not cause interference to PUs and QoS for SUs is still at an acceptable level.

\section{ACKNOWLEDGMENT}

The authors would like to acknowledge Atso Hekkala and Miia Mustonen for the help with simulations.

\section{REFERENCES}

[1] S. Haykin, "Cognitive radio: Brain-empowered wireless communications," IEEE Journal on Selected Areas in Communications, vol. 23, no. 2, pp. 201-220, February 2005.

[2] D. Cabric, S. M. Mishra, and R. W. Brodersen, "Implementation issues in spectrum sensing for cognitive radios," in Proc. Asilomar Conference, pp. 772-776, Nov. 2004.

[3] B. V. Veen and K. M. Buckley, "Beamforming: A versatile approach to spatial filtering," IEEE ASSP Magazine, vol. 5 no. 2, pp. 4-24, April 1988.

[4] M. Matinmikko, M. Mustonen, M. Höyhtyä, T. Rauma, H. Sarvanko, and A. Mämmelä, "Distributed and directional spectrum occupancy measurements in the $2.4 \mathrm{GHz}$ ISM band," in Proc. Seventh International Symposium on Wireless Communication Systems (IEEE ISWCS 2010), York, UK, 2010.

[5] L. Zhang, Y.-C. Liang, and Y. Xin, "Joint beamforming and power allocation for multiple access channels in cognitive radio networks," IEEE Journal on Selected Areas in Communications, vol. 26, no. 1, pp. 38-51, Jan. 2008.

[6] M. H. Islam, Y. Liang, and A. T. Hoang, "Joint power control and beamforming for cognitive radio networks," IEEE Trans. Wireless Commun., vol. 7, pp. 2415-2419, July 2008.

[7] R. Zhang, F. Gao, and Y.-C. Liang, "Cognitive beamforming made practical: Effective interference channel and learning-throughput tradeoff," in Proc. IEEE Workshop on SPAWC '09, pp. 588-592, June 2009.

[8] R. Zhang and Y. C. Liang, "Exploiting multi-antennas for opportunistic spectrum sharing in cognitive radio networks," IEEE J. Select. Topics Signal Processing, vol. 2, pp. 88-102, Feb. 2008.

[9] F. Gao, R. Zhang, Y.-C. Liang, and X. Wang, "Multi-antenna cognitive radio systems: Environmental learning and channel training," in Proc. IEEE ICASSP 2009, pp. 2329-2332, April 2009.

[10] G. Zheng, S. Ma, K.-K. Wong, and T.-S. Ng, "Robust beamforming in cognitive radio," IEEE Trans. Wireless Commun, vol. 9, no. 2, pp. 570576 , February 2010.

[11] S. Wang, Q. Liu, and Z. Zhou, "A cooperative cognitive radio network using smart antenna," in Proc. IEEE ICCTA 2009, pp. 450-456, Oct. 2009.

[12] Z. Zhuang, S. Sohn, J. Gu, and J. Kim, "An interference mitigated and power efficient adaptive beamforming algorithm in cognitive radio," in Proc. IEEE WiCom 2009, Sept. 2009.

[13] P. Gupta and P. R. Kumar, "The capacity of wireless networks," IEEE Trans. Inform. Theory, vol. 46, no. 2, pp. 388-404, Mar. 2000.

[14] K.-L. Du and W. H. Mow, "Affordable cyclostationarity-based spectrum sensing for cognitive radio with smart antennas," IEEE Transactions on Vehicular Technology, vol. 59, no. 4, pp. 1877-1886, May 2010

[15] A.Taherpour, N. Nasiri-Kenari, and S. Gazor, "Multiple antenna spectrum sensing in cognitive radios," IEEE Transactions on Wireless Communications, vol. 9, no. 2, pp. 814-823, Feb. 2010

[16] H. Urkowitz, "Energy detection of unknown deterministic signals," Proc. IEEE, vol. 55, no. 4, pp. 523-531, Apr. 1967.

[17] F. F. Digham, M.-S. Alouini, and M. K. Simon, "On the energy detection of unknown signals over fading channels," in Proc. IEEE International Conference on Communications, vol. 5, Anchorage, AK, Mar. 11-15, 2003, pp. 3575-3579.

[18] H. Sarvanko et al., "Cooperative and noncooperative spectrum sensing techniques using Welch's periodogram in cognitive radios," in Proc. First International Workshop on Cognitive Radio and Advanced Spectrum Management, Feb. 2008.

[19] J. Litva and T. Kwok-Yeung Lo, Digital Beamforming in Wireless Communications. Boston, MA: Artech House, 1996. 